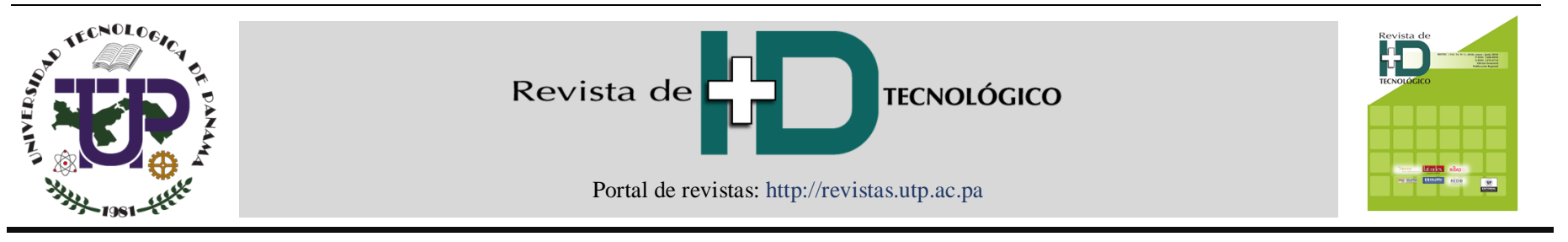

\title{
Control de seguimiento para un aerodeslizador no tripulado por medio de un único actuador
}

\section{Trajectory tracking control of an unmanned hovercraft by means of A single actuator}

\author{
Ricardo De Levante ${ }^{1 *}$, Deyka García ${ }^{2}$ \\ ${ }^{1}$ Facultad de Ingeniería Eléctrica, Universidad Tecnológica de Panamá, Panamá \\ ${ }^{2}$ Facultad de Ingeniería Mecánica, Universidad Tecnológica de Panamá, Panamá \\ *Autor de correspondencia: ricardo.delevante@utp.ac.pa
}

\begin{abstract}
RESUMEN- Se presenta el desarrollo de un regulador cuadrático lineal como controlador para la navegación de un sistema no holonómico y subactuado: un aerodeslizador no tripulado. En este trabajo se utiliza un modelo simplificado del aerodeslizador derivado del modelo general de vehículo de superficie. Este modelo considera tres grados de libertad para el vehículo, dos de estos grados son completamente actuados; además, se añade al grado no actuado un coeficiente que representa las fuerzas de arrastre que se oponen al movimiento del vehículo. Aprovechando que este modelo del aerodeslizador es diferencialmente plano según un marco inercial de referencia, se diseña una trayectoria circular como consigna. Se verifica la controlabilidad del vehículo según el modelo encontrado, que es controlable siempre que la velocidad angular a la que gira sea distinta de cero. Este discernimiento se aprovecha, luego de asumir que la velocidad angular del vehículo es constante, para realizar un controlador que utiliza un único actuador para seguir la trayectoria deseada. Se presenta la ley de control propuesta, las ganancias de la ley de control son determinadas por un regulador cuadrático lineal. El regulador cuadrático lineal se sintoniza utilizando como punto de partida la Regla de Bryson, y se mejora mediante ensayo y error. Se finaliza con una serie de simulaciones realizadas en el software MATLAB probando que, aunque el controlador es poco eficiente en su respuesta, logra seguir la trayectoria circular deseada.
\end{abstract}

\section{Palabras clave-Aerodeslizador, controlador, no holonómico, regulador cuadrático lineal, sistema subactuado.}

\begin{abstract}
This paper presents the development of a linear quadratic regulator for the navigational control of an underactuated and nonholonomic system: the unmanned hovercraft; a simplified model of the hovercraft derived from the general model for surface vehicles is used for this end. This model considers three degrees of freedom for the hovercraft; two of them are completely actuated. A coefficient representing the drag forces perceived by the vehicle is added in the direction of the unactuated degree. It is found while verifying the controllability of the hovercraft that the system is controllable as long as the angular velocity is not cero. Taking advantage that this model of the hovercraft is differentially flat with respect to an inertial reference frame a circular trajectory is designed as the reference for the controller. After assuming that the angular velocity of the hovercraft is constant, these properties are used to design a controller that uses a single actuator to follow the reference trajectory. This paper proposes a control law that uses a linear quadratic regulator to determine its gains. The regulator is tuned by trial and error, starting from Bryson's Rule. This paper finishes with a series of simulations programmed on the software package MATLAB proving that, although the controller is not efficient, it follows the trajectory by means of a single actuation signal as expected.
\end{abstract}

Keywords-Hovercraft, Controller, not holonomic, linear quadratic regulator, under-system.

\section{Introducción}

La agricultura moderna se divide en dos grandes ramas, la agricultura convencional y la agricultura de precisión. La agricultura de precisión se define, para este estudio, de la siguiente manera: "un sistema de manejo basado en información y tecnología que es específico al sitio y usa al menos una de las siguientes fuentes de datos: suelos, cultivos, nutrientes, pestes, humedad, o rendimiento, para lograr una óptima rentabilidad, sostenibilidad y protección del ambiente" (traducido y adaptado de [1]).
La agricultura de precisión tiene el objetivo de aprovechar al máximo los insumos tomando en cuenta las irregularidades de los campos sembrados, las necesidades específicas de ciertos grupos de plantas y otras características de los sembradíos.

La agricultura de precisión prefiere equipos que entren en contacto próximo con plantas o suelos, asegurando que los insumos sean aprovechados al máximo. Se busca en la agricultura de precisión limitar el uso de avionetas para regadío, pues los químicos se dispersan, impidiendo una distribución eficiente. Uno de los mecanismos implementados, consiste en usar

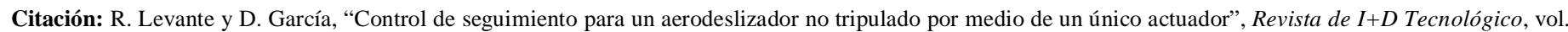
15, no. 1, pp. (38-48), 2019.

Tipo de artículo: Original. Recibido: 14 de agosto de 2018. Recibido con correcciones: 17 de agosto de 2018 . Aceptado: 19 de noviembre de 2019.

DOI https://doi.org/10.33412/idt.v15.1.2096

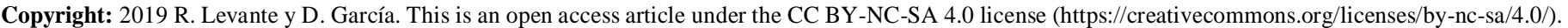


personas, que carguen los químicos pesticidas o similares y las suministren directamente a las plantas. Ya que un suministro directo a la planta de productos químicos, presenta un riesgo de salud, para operarios [2] se propone como alternativa introducir vehículos no tripulados y automáticos para realizar las funciones de la agricultura de precisión.

El aerodeslizador es un vehículo de sustentación de aire, capaz de flotar sobre cualquier superficie continua. $\mathrm{Su}$ extensión es delimitada por una falda, o manta flexible, al moverse sobre plantas las dobla sin quebrarlas por lo que un aerodeslizador se puede utilizar para monitorear y regar ciertos sembradíos. Un uso aún más interesante de los aerodeslizadores para agricultura consiste en la irrigación de suelos lodosos; este uso se conoce al menos desde los años 60 , cómo se evidencia por el vehículo Hover Rover fabricado por Vickers-Armstrong Limited. El Hover Rover mostrado en la figura 2 consiste en un tractor al cual se le ha añadido el sistema de inflado y falda para convertirlo en un aerodeslizador. Normalmente, un tractor se atascaría en terreno lodoso, haciendo imposible el tratamiento del suelo hasta que la tierra seque, añadiendo semanas al ciclo agrícola. Al bajarla falda hasta la tierra y llenarla de aire, el peso sobre las ruedas disminuye y el vehículo se puede hacer deslizar sobre el terreno lodoso impulsado ya sea por impulso de aire o por la acción de las ruedas [3]. De este modo un aerodeslizador puede ser usado en agricultura de precisión y se hace patente la necesidad de nuevos estudios en la aplicabilidad de aerodeslizadores para agricultura.

El aerodeslizador, como generalmente se diseña, consta de un sistema de inflado, que llena la falda de aire y crea la sustentación para el vehículo; incluye un sistema de propulsión que se suele unir al sistema de inflado para minimizar la cantidad de ventiladores necesarios; en específico para el caso de un aerodeslizador automático y no tripulado consta también de un sistema de control, que incluye una red de sensores. El sistema de propulsión es también el responsable del direccionamiento del vehículo por lo que es este sistema el objeto de control para navegación. Un aerodeslizador pequeño diseñado y construido por los autores cómo parte de un estudio relacionado se muestra en la figura $\mathbf{1}$

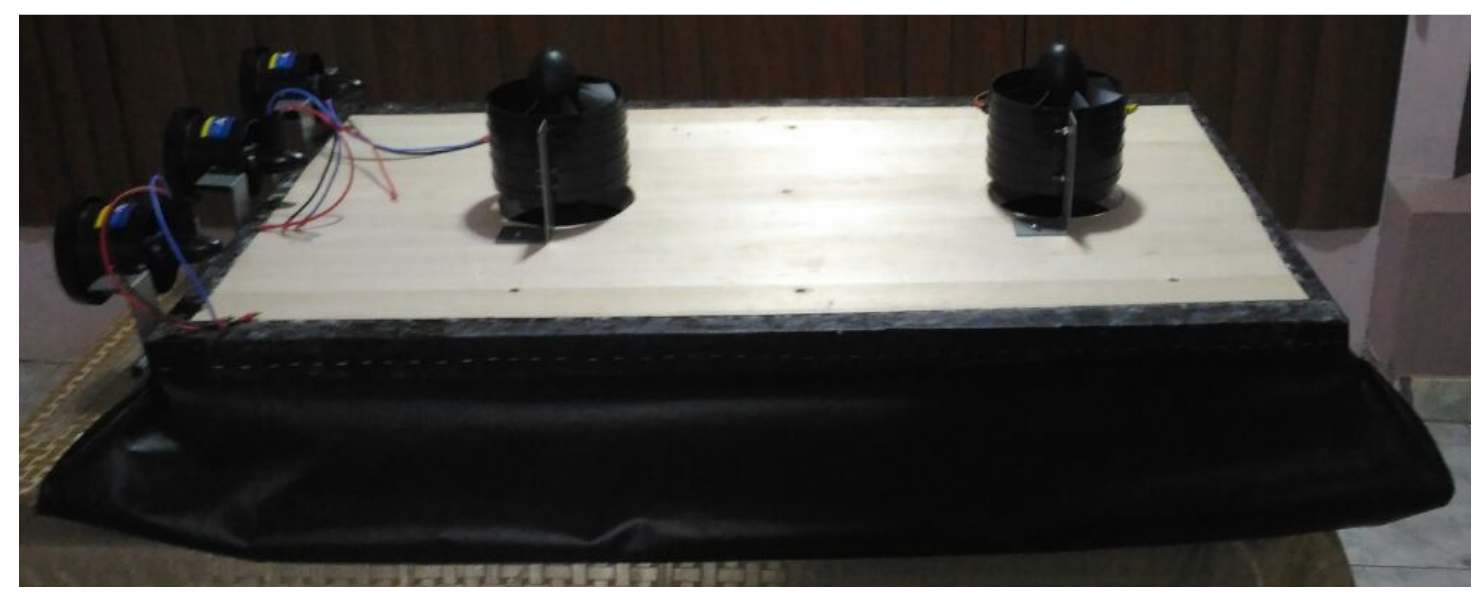

Figura 1. Vehículo aerodeslizador construido por los autores. Hecho de madera balsa con una falda de poliéster recubierta con PVC. Los dos ventiladores sobre la cubierta del vehículo inflan la falda. Los tres ventiladores montados en la popa del vehículo impulsan el vehículo. Si el ventilador de babor genera un impulso mayor que el ventilador de estribor, el aerodeslizador giraría en sentido contrario.

El desafío de controlar un aerodeslizador surge de su dinámica. El vehículo es no holonómico, por lo que su posición no se puede determinar completamente por su cinemática. Implica también controlar un aerodeslizador subactuado, por lo que hay grados de libertad sin actuación.
Un sistema no holonómico es aquel sistema cuyos estados dependen del camino tomado. En un sistema como este, se pueden identificar una serie de parámetros que varían conforme el sistema evoluciona, y aunque estos parámetros vuelvan a los del punto de partida, el estado del sistema puede no ser el mismo. Una definición 
un poco más precisa de un sistema no holonómico se refiere a aquel sistema que presenta ecuaciones de restricción en las velocidades del sistema, diferenciándose de un sistema holonómico que solo presenta restricciones en las posiciones. Las restricciones de un sistema no holonómico se presentan como relaciones diferenciales no integrables; se encuentra, además, que el número de grados de libertad de este sistema es menor al número de coordenadas independientes en una cantidad igual al número de estas restricciones. Finalmente, en un sistema no holonómico, el movimiento entre posiciones infinitesimalmente vecinas, puede ser imposible, añadiendo una dificultad adicional al planeamiento de la trayectoria de un sistema como este [4].

Un sistema subactuado es aquel en el cual hay una menor cantidad de actuadores por grado de libertad que grados de libertad. Un sistema con tres grados de libertad que únicamente posee actuación en dos grados, es un sistema subactuado. Un sistema subactuado también se puede entender como aquel sistema que no puede acelerarse instantáneamente por sus actuadores en alguna dirección arbitraria. Como una consecuencia de esto, los sistemas subactuados no pueden ser dirigidos a seguir trayectorias arbitrarias [5]. Los sistemas subactuados contrastan con los sistemas completamente actuados, donde cada grado de libertad posee actuación y es posible acelerar el sistema instantáneamente a una dirección arbitraria.

El diseño de sistemas de control para aerodeslizadores es un campo de trabajo activo en la actualidad [6], con enfoques a seguimientos de trayectoria y navegación también en la literatura [7], incluyendo modelos de aerodeslizadores subactuados. Las soluciones que se suelen encontrar en la literatura hacen uso de al menos dos actuadores para controlar el vehículo, este estudio busca abrir la discusión hacia el diseño de controladores de sistemas subactuados con un mínimo de actuadores, en este caso un único actuador; logrando este objetivo aprovechando ciertas características propias de la dinámica de los aerodeslizadores.

Este trabajo se realiza como seguimiento al trabajo realizado por García et al., quien utilizó el método directo de Lyapunov [6] y formuló un diseño para la estabilización de un aerodeslizador [8]. En el trabajo de García et al., se diseña un controlador para estabilizar la posición de un aerodeslizador, de tal forma que permanezca indefinidamente en su posición de consigna sin deslizarse o girar. En este trabajo se aprovecha la baja resistencia dinámica del aerodeslizador que le permite mantener algún giro que se le haya sido impartido en la dirección de guiñada para realizar el seguimiento de una trayectoria circular únicamente con un actuador actuando en la dirección de proa-popa.

En este trabajo se trata el diseño de un controlador basado en reguladores cuadráticos para navegar un vehículo aerodeslizador. Se presenta primero el modelo del aerodeslizador, el cual está basado en los vehículos de superficie, como botes. Al analizar el modelo del vehículo se nota que el mismo es no holonómico y subactuado. Atendiendo a estas limitaciones del vehículo se introduce un difeomorfismo para tratar el hecho de que sea no holonómico y se encuentra una trayectoria circular como solución para tratar el problema de la subactuación. Se nota que, debido en parte a las consideraciones tomadas, el seguimiento de la trayectoria es lograda con una sola señal de actuación.

Ya que el aerodeslizador debe ser autónomo para eliminar la necesidad de un operario, este debe ser capaz de seguir automáticamente alguna trayectoria de trabajo sobre la cual realizar su acción agrícola. Como primer paso se busca la navegación a una trayectoria circular, aprovechando también el hecho de que, a partir de una secuencia de trayectorias circulares, es posible construir trayectorias más complejas.

Este trabajo propone un método para lograr que un aerodeslizador siga una trayectoria circular utilizando un único actuador, siendo este el aporte al estado del arte. Se presentan simulaciones realizadas con el software MATLAB del controlador propuesto ante diversas condiciones iniciales como evidencia de su funcionamiento.

El trabajo presentado aquí fue realizado con fondos provistos por la Secretaria Nacional de Ciencia, Tecnología e Innovación (SENACYT) de Panamá, por medio del Sistema Nacional de Investigación (SNI).

\section{Modelado dinámico del aerodeslizador}

La dinámica de un aerodeslizador se puede modelar cómo un vehículo de superficie [9] al considerar al aerodeslizador como un vehículo que se sostiene sobre una capa de aire, y que flota de manera similar a como lo haría un bote sobre el agua. En este modelo se utilizan dos marcos de referencia para describir la dinámica del 
vehículo: uno inercial y otro no-inercial, este último sobre el vehículo. El movimiento solamente se considera en el plano x-y, esto es superficial; no se considera relevante que el vehículo cambie su altura. Aunque el cabeceo de la nave sí tiene un aporte al movimiento en el plano del vehículo, principalmente debido a que los escapes de aire modifican la dinámica del sistema ya sea añadiendo fuerzas o cambiando el peso del vehículo, un balance correcto del sistema de inflado y un cuidado con la superficie sobre la que se navega, minimiza estos efectos en la práctica.

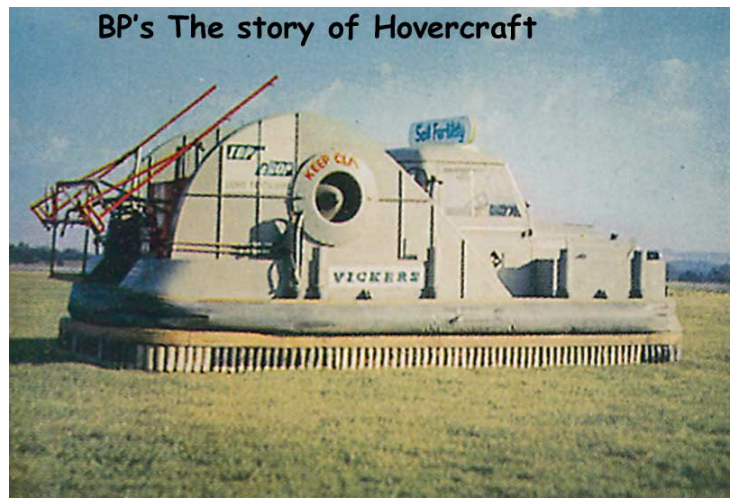

Figura 2. Vehículo Aerodeslizador Hover Rover modelo del fabricante inglés Vickers-Armstrong Limited tomado de [3]. El vehículo se utiliza para irrigación de suelos, existen videos de su operación en internet ${ }^{1}$. Fotografía usada con permiso.

En la figura 3 se muestra el modelo simplificado del aerodeslizador adaptado para este estudio de [6]. Para este modelo se considera un sistema de referencia inercial local. De este modo se desprecian las fuerzas de Coriolis inducidas por la rotación de la Tierra; también, se considera a la Tierra localmente plana.

Refiriéndose a la figura $3, x, y, \psi$ representan la posición generalizada y la orientación según el marco de referencia inercial. El marco sobre el vehículo considera dos ejes. Uno de los ejes, el eje transversal, en la dirección babor-estribor, según el cual se mide la velocidad de abatimiento, indicada con $v$. El otro eje, el

\footnotetext{
${ }^{1}$ Archiveros virtuales han recabado videos de la operación de este vehículo para la irrigación de tierras para cultivos como: https://www.britishpathe.com/video/hover-rover
}

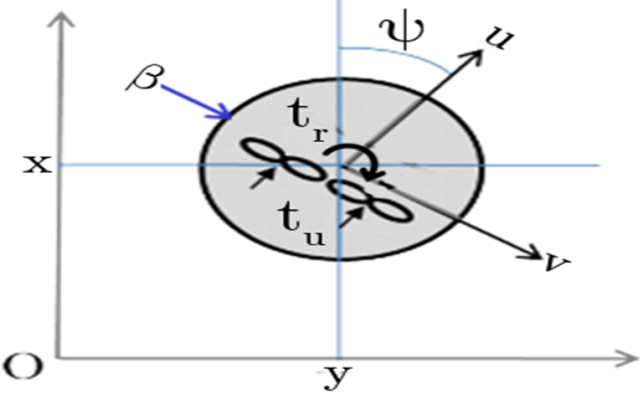

Figura 3. Modelo del aerodeslizador, adaptado de [6].

longitudinal, en la dirección popa-proa, según el cual se mide la velocidad de avance $u$. En el eje longitudinal de la nave se han montado al menos dos ventiladores, que proveen impulso a la nave. Funcionando como actuadores del sistema de control de la nave; la fuerza que aportan al movimiento se denota con $\tau_{u}$. Estos mismos ventiladores se pueden hacer operar y disponer en una variedad de configuraciones; es posible entonces adicionar al sistema un segundo actuador al configurar los ventiladores como un diferencial de torsión, por ejemplo. Este segundo actuador proveerá un momento de torsión para hacer girar la nave actuando sobre el ángulo de guiñada. El momento de torsión provisto de esta manera se indica por $\tau_{r}$. Al modelo se añade un elemento a veces despreciado en la literatura pero que se puede encontrar en trabajos como [7], un coeficiente que representa el efecto de las fuerzas de arrastre en la dirección estribor-babor, $\beta$; este coeficiente es constante y depende del diseño del vehículo y su entorno de trabajo; es posible dimensionarlo mediante pruebas de laboratorio. Al analizar este modelo siguiendo un desarrollo similar al descrito en [6] encontramos la siguiente dinámica del vehículo. Se recalca que todos los términos, excepto $\beta$ y aquellos que representan funciones trigonométricas, son variables

$$
\begin{aligned}
& \dot{\mathrm{x}}=u \cos (\psi)-v \operatorname{sen}(\psi) \\
& \dot{y}=u \operatorname{sen}(\psi)+v \cos (\psi) \\
& \dot{\psi}=r \\
& \dot{u}=v r+\tau_{u} \\
& \dot{v}=-u r-\beta v \\
& \dot{r}=\tau_{r}
\end{aligned}
$$


Donde,

$u$ es la velocidad $(\mathrm{m} / \mathrm{s})$ hacia proa en el eje longitudinal de la nave,

$v$ es la velocidad $(\mathrm{m} / \mathrm{s})$ hacia estribor en el eje transversal,

$\psi$ es el ángulo de guiñada de la nave (rad),

$r$ es la velocidad angular ( $\mathrm{rad} / \mathrm{s}$ ) en el ángulo de guiñada de la nave, se omite de la figura 3 por espacio,

$x$ es la posición del vehículo según el eje vertical de un sistema inercial de referencia (m),

$y$ es la posición del vehículo según el eje horizontal de un sistema inercial de referencia(m),

$\beta$ es un coeficiente (1/s) que representa el arrastre del vehículo en la dirección estribor-babor,

$\tau_{u}$ es la fuerza aplicada en el eje longitudinal de la nave $(\mathrm{N})$,

$\tau_{r}$ es el torque aplicado a través del eje vertical de la nave, en la dirección del ángulo de guiñada (N/s).

Para obtener este modelo se han aplicado ciertas condiciones a la dinámica del vehículo, las mismas se suelen encontrar en la literatura [9] como aproximaciones para estos sistemas. Se considera que la forma del vehículo es un disco, que los ventiladores se ubican en el centro de masa del vehículo, que la matriz de inercia del vehículo, según el modelo de vehículos de superficie (ver [7]), es diagonal e igual a la matriz identidad. Se considera que solamente existe arrastre aerodinámico y que este solamente actúa en el eje de estribor a babor de la nave, en la dirección de $v$. También, por el diseño del vehículo no hay actuador en este eje, por lo que no hay fuerza externa en su ecuación. También se considera que la aceleración angular en el ángulo de guiñada es directamente controlable por el torque aplicado, aproximación que es posible hacer al considerar despreciables los efectos del arrastre aerodinámico en este movimiento. La baja resistencia al movimiento del aerodeslizador se modela aquí considerando que la actuación en el ángulo de guiñada controla completamente la aceleración en esta dirección. Esto se aprovecha para el diseño del controlador de la siguiente manera: el aerodeslizador permanece en movimiento a una velocidad angular constante, definida por el impulso inicial, en la ausencia de fuerzas no-conservativas.

\section{Planeación de la trayectoria}

El sistema del aerodeslizador que se muestra en la ecuación (1) es diferencialmente plano con respecto a las salidas $x$ y $y$ [7]. Esto implica que todas las variables del sistema se pueden expresar como funciones de las variables de salida, $x$ y $y$, y sus derivadas. Esto hace posible expresar el ángulo de guiñada en términos de las variables de salida y, a partir de este resultado, encontrar una trayectoria posible para este sistema tomando en cuenta sus limitaciones. Siguiendo el procedimiento detallado en [7], el ángulo de guiñada se puede expresar en términos de estas variables como se muestra en la ecuación (2). El término $\beta$ es constante.

$$
\psi=\arctan \left(\frac{\ddot{y}+\beta \dot{y}}{\ddot{x}+\beta \dot{x}}\right)
$$

A partir de las primeras dos líneas de la ecuación (1) se puede encontrar otro resultado de utilidad: expresiones para $u \mathrm{y}_{v}$ en términos de las variables de salida.

$$
\begin{aligned}
& v=\dot{y} \cos (\psi)-\dot{x} \operatorname{sen}(\psi) \\
& u=\dot{y} \operatorname{sen}(\psi)+\dot{x} \cos (\psi)
\end{aligned}
$$

Tomando la ecuación (2) junto con la ecuación (3) se encuentran el conjunto de ecuaciones que deben solucionarse para encontrar una trayectoria posible para el aerodeslizador, expresada en la ecuación (4).

$$
\begin{aligned}
& \psi_{d}=\arctan \left(\frac{\ddot{y}_{d}+\beta \dot{y}_{d}}{\ddot{x}_{d}+\beta \dot{x}_{d}}\right) \\
& v_{d}=\dot{y}_{d} \cos \left(\psi_{d}\right)-\dot{x}_{d} \operatorname{sen}\left(\psi_{d}\right) \\
& u_{d}=\dot{y}_{d} \operatorname{sen}\left(\psi_{d}\right)+\dot{x}_{d} \cos \left(\psi_{d}\right) \\
& r_{d}=\dot{\psi}_{d}
\end{aligned}
$$

En la ecuación (4) se usan las mismas letras que en la ecuación (1) para representar las mismas variables. A cada letra se añade el subíndice $d$, de este modo se indica que esta representa las características de la trayectoria deseada, también llamada de referencia o consigna.

Al elegir una trayectoria $y_{d}$ y $x_{d}$ para que el vehículo siga, esta debe ser una solución del sistema de ecuaciones (4). Se elige una trayectoria con forma de círculo y el sistema se resuelve a la ecuación (5).

$$
\begin{aligned}
x_{d} & =p \cos (w t) \\
y_{d} & =p \sin (w t) \\
\psi_{d} & =w t+\arctan \left(\frac{\beta}{w}\right) \\
v_{d} & =p w \cos \left(\arctan \left(\frac{\beta}{w}\right)\right) \\
u_{d} & =-p w \sin \left(\arctan \left(\frac{\beta}{w}\right)\right) \\
r_{d} & =w
\end{aligned}
$$

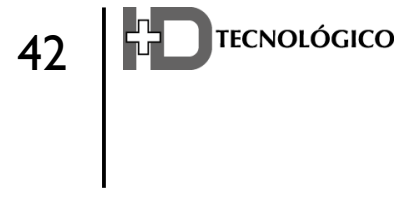


Para la ecuación (5) cual,

$p$ es el radio del círculo,

${ }_{w}$ es la frecuencia de oscilación,

$t$ es el tiempo,

y las unidades son definidas según las dimensiones del sistema. Un resultado interesante de esta elección surge debido a la consideración de que el ángulo de guiñada es directamente controlable por lo que se puede, seguir completamente $r_{d}$, ya que esta es constante, la acción del actuador se extingue con el tiempo y la navegación del sistema se logra únicamente con el actuador de avance. Así si se imparte un impulso de torsión al vehículo que lo haga girar constantemente a la frecuencia de oscilación ${ }_{w}$, este seguirá la trayectoria circular únicamente con los actuadores de avance. Este es un resultado que solo se ha podido verificar para trayectorias circulares en este estudio.

\section{Controlabilidad del aerodeslizador}

Para describir el movimiento del sistema en el sistema de referencia inercial con respecto al plano no inercial se aplica una transformación, que es un difeomorfismo, y se muestra en la ecuación (6) [9].

$$
\begin{aligned}
& Z_{1}=\cos (\psi) x+\sin (\psi) y \\
& Z_{2}=-\sin (\psi) x+\cos (\psi) y \\
& Z_{3}=\psi
\end{aligned}
$$

Al aplicar la ecuación (6) en conjunto con la ecuación (1) el sistema del aerodeslizador se reduce de la siguiente manera.

$$
\begin{aligned}
& \dot{Z}_{1}=u+Z_{2} r \\
& \dot{Z}_{2}=v-Z_{1} r \\
& \dot{u}=v r+\tau_{u} \\
& \dot{v}=-u r-\beta v
\end{aligned}
$$

donde se han despreciado las ecuaciones que corresponden a la actuación en el ángulo de guiñada, ya que se está considerando que este grado es directamente controlable. Esto implica que se desprecie la derivada de la variable temporal $r$, que para la trayectoria que se usará tendrá un valor nulo. La ecuación (7) se reescribe en representación de espacio de estados para aplicar el criterio de controlabilidad, esta formulación se aprecia en la ecuación (8). En la ecuación (8) A es la matriz del sistema, $\mathrm{B}$ es la matriz de entrada y $q$ es el vector de estados y $U$ es el vector de entradas del sistema, que para esta aplicación solo tiene una entrada: $\tau_{u}$; también se presenta en la ecuación (8) la matriz de salida.

$$
\begin{gathered}
{\left[\begin{array}{c}
\dot{Z}_{1} \\
\dot{Z}_{2} \\
\dot{u} \\
\dot{v}
\end{array}\right]=\left[\begin{array}{cccc}
0 & r & 1 & 0 \\
-r & 0 & 0 & 1 \\
0 & 0 & 0 & r \\
0 & 0 & -r & -\beta
\end{array}\right]\left[\begin{array}{c}
Z_{1} \\
Z_{2} \\
u \\
v
\end{array}\right]+\left[\begin{array}{l}
0 \\
0 \\
1 \\
0
\end{array}\right]\left[\begin{array}{c}
\tau_{u} \\
]
\end{array}\right]=A q+B U} \\
{\left[\begin{array}{c}
Z_{1 o} \\
Z_{2 o} \\
u_{o} \\
v_{o}
\end{array}\right]=\left[\begin{array}{llll}
1 & 0 & 0 & 0 \\
0 & 1 & 0 & 0 \\
0 & 0 & 1 & 0 \\
0 & 0 & 0 & 1
\end{array}\right]\left[\begin{array}{c}
Z_{1} \\
Z_{2} \\
u \\
v
\end{array}\right]}
\end{gathered}
$$

Se requiere que al formar la matriz de controlabilidad del sistema esta sea de rango completo para que el sistema sea controlable.

$$
R_{C}=\left[\begin{array}{cccc}
1 & 0 & -r^{2} & \beta r^{2} \\
0 & -r & \beta r & -r\left(\beta^{2}-r^{2}\right) \\
0 & 1 & 0 & -3 r^{2} \\
0 & 0 & -2 r & \beta r
\end{array}\right]
$$

La ecuación (9) es la matriz de controlabilidad del sistema. Esta matriz es de rango completo siempre que $r$, la velocidad angular del vehículo en el ángulo de guiñada, sea distinta de cero. Para el caso de la trayectoria circular, $r$ será constante y distinta de cero, por lo que el sistema es controlable para este caso.

\section{Control por regulación cuadrática lineal}

Se utiliza un regulador cuadrático lineal para construir un controlador para el aerodeslizador y así navegar el aerodeslizador por la trayectoria circular. Haciendo referencia a la ecuación (10) tenemos:

$$
\begin{gathered}
\dot{q}=A q+B U \\
U=-K q \\
J\left(t, t_{f}\right)=\int_{t}^{t_{f}}\left(q^{T} Q q+u^{T} R u\right) d \tau
\end{gathered}
$$

donde,

$q$ es el vector de estados del sistema,

${ }_{A}$ es la matriz de la dinámica de sistema,

${ }_{B}$ es la matriz de actuación del sistema,

$U$ es el vector de controles del sistema,

$K$ es una matriz de ganancias,

$Q$ es la matriz de peso de los estados,

$R$ es la matriz de costo de control.

Prosigue un resumen de reguladores cuadráticos lineales [10]. Para un sistema como el descrito en (10) se puede definir un controlador proporcional como el que se 
muestra en (11), donde la matriz $K$ se puede definir como la solución a la ecuación de Riccati que minimiza la función de costo definida en la ecuación (12).

Para que exista la matriz $K$ es necesario que las matrices $R$ y $Q$ en la ecuación (12) cumplan ciertas condiciones. $R$ debe ser una matriz simétrica positiva definida y $Q$ debe ser simétrica y al menos semipositiva definida. Las matrices $R$ y $Q$ son dimensionadas por el diseñador y su elección es uno de los desafíos principales del diseño de un controlador por regulación cuadrática lineal. Las matrices $R$ y $Q$ son usadas para definir la importancia que tiene para la función de costo $J$ la magnitud de las entradas de control o de los estados; así si un diseñador quiere minimizar la energía que consumen las entradas de control es solo necesario que se elija una matriz $R$ cuya influencia en la función de costo sea mayor que la matriz $Q$. Es posible utilizar la Regla de Bryson [11] como punto de partida para el dimensionamiento de estas matrices, no obstante su afinamiento se realiza por prueba y error.

A continuación, se definirá el error $e$ del sistema de control. Primero es necesario construir la trayectoria deseada, un círculo, en términos de la transformación que se realizó en la sección 4. Usando la ecuación (5) y el modelo que se presenta en la ecuación (6), se encuentra la trayectoria deseada y se presenta en la ecuación (13).

Donde el subíndice $d$ indica que se trata de valores deseados o de referencia. Se recalca que de interés para este estudio son las salidas definidas en la ecuación (10), en especial las dos variables $Z_{1}$ y $Z_{2}$, de las cuales es posible reconstruir la trayectoria que sigue el vehículo. Ahora se procede a comparar la trayectoria deseada con el vector de estados, el vector resultante es el error del sistema, e. Se presenta en la ecuación (14). Donde $Z_{1}, Z_{2}$, $u$ y $v$ son los valores actuales de las variables de estado durante operación, reportados, por ejemplo, por una red de sensores de posición y aceleración.

$$
\begin{gathered}
Z_{1 d}=\cos \left(w t+\arctan \left(\frac{\beta}{w}\right)\right) p \cos (w t) \\
\quad+\sin \left(w t+\arctan \left(\frac{\beta}{w}\right)\right) p \sin (w t) \\
Z_{2 d}=-\sin \left(w t+\arctan \left(\frac{\beta}{w}\right)\right) p \cos (w t) \\
\quad+\cos \left(w t+\arctan \left(\frac{\beta}{w}\right)\right) p \sin (w t) \\
v_{d}=p w \cos \left(\arctan \left(\frac{\beta}{w}\right)\right) \\
u_{d}=-p w \sin \left(\arctan \left(\frac{\beta}{w}\right)\right)
\end{gathered}
$$

$$
e=\left[\begin{array}{c}
Z_{1 d^{-Z}} 1 \\
Z_{2 d^{-Z}} \\
u_{d}-u \\
v_{d}-v
\end{array}\right]
$$

Tomando el controlador sugerido en [10] que se muestra en la ecuación (11) como referencia, se propone una ley de control para el sistema del aerodeslizador definido en la ecuación (10). La ley de control propuesta para el seguimiento de trayectoria se define por la ecuación (15). Los términos mantienen su significado de la ecuación (11) y la ecuación (14).

$$
U=-K e
$$

donde,

$e$ es la diferencia entre el vector de estados deseados y el vector de estados actuales o medidos.

\section{Simulación}

Se usa el software MATLAB para simular el sistema dinámico y la actuación del controlador sobre él. Se escoge MATLAB ya que incluye funciones para solucionar la ecuación algebraica de Riccati y por la facilidad de escribir un programa y poder cambiar las condiciones iniciales, facilitando el encontrar la respuesta del sistema frente a diferentes condiciones.

En MATLAB hay dos funciones que son de especial utilidad para simular el desarrollo de este estudio. La primera función a mencionar, es la función initial; la cual, a partir de un sistema dinámico representado en espacio de estados obtiene la respuesta natural para cualquier condición inicial. La segunda función de utilidad es lqr esta función se invoca para diseñar, a partir de la dinámica de un sistema y los parámetros de la función de costo, un regulador cuadrático lineal; esto es, al invocar esta función se obtiene el valor de la ganancia $K$. En general para el diseño de las simulaciones se sigue un procedimiento similar al propuesto en [10].

Es necesario definir en MATLAB la dinámica del sistema, haciendo referencia a la ecuación (10), la matriz A se rellena con los valores de la consigna, recordando que esta matriz solo depende de la velocidad de giro del vehículo y en nuestro tratamiento esta es constante; la matriz B y todos los parámetros se normalizan dividiendo entre la masa del vehículo. El vehículo de simulación se considera un aerodeslizador pequeño de unos $30 \mathrm{~kg}$, es con este valor que se normaliza la matriz B y el resto del 
sistema. Se presenta en la ecuación (16) las matrices que se usaron en simulación.

$$
\left[\begin{array}{c}
\dot{Z}_{1} \\
\dot{Z}_{2} \\
\dot{u} \\
\dot{v}
\end{array}\right]=\left[\begin{array}{cccc}
0 & \frac{w}{30} & 1 & 0 \\
-\frac{w}{30} & 0 & 0 & 1 \\
0 & 0 & 0 & \frac{w}{30} \\
0 & 0 & -\frac{w}{30} & \frac{347}{3000}
\end{array}\right]\left[\begin{array}{c}
Z_{1} \\
z_{2} \\
u \\
v
\end{array}\right]+\left[\begin{array}{c}
0 \\
0 \\
\frac{1}{30} \\
0
\end{array}\right]\left[\tau_{u}\right]
$$

Para elegir las matrices de costo $Q$ y $R$ se inicia usando la Regla de Bryson. En la Regla de Bryson se rellena únicamente la diagonal principal de cada matriz escogiendo valores máximos que dependen de parámetros como el tiempo de muestreo y la fineza de los mecanismos de medición, luego los valores de la matriz son modificados hasta que la respuesta llegue a estar dentro de algún rango de valores deseados. En la tabla 1

se muestra el parámetro adimensional $u_{1 \max }$, este corresponde con el valor inicial según la Regla de Bryson para la matriz $R$, que solo tiene un valor. Los valores $x_{1 \text { max }}$ hasta $x_{4 \max }$, son parámetros adimensionales utilizados en la sintonización de la matriz $Q$. Ellos corresponden con los valores de la matriz $Q$, en orden cada uno se relaciona a una de las cuatro variables de estado del sistema. Las matrices $Q$ y $R$ en sus versiones para los resultados de estas simulaciones se muestran en la ecuación (17).

$$
\begin{gathered}
Q=\left[\begin{array}{cccc}
1 \times 10^{5} & 0 & 0 & 0 \\
0 & 1 \times 10^{3} & 0 & 0 \\
0 & 0 & 1 \times 10^{11} & 0 \\
0 & 0 & 0 & 1 \times 10^{7}
\end{array}\right] \\
R=\left[7 \times 10^{6}\right]
\end{gathered}
$$

La tabla 1 muestra los valores escogidos para realizar la simulación del aerodeslizador. Los primeros dos valores de la columna de la izquierda corresponden a la trayectoria a seguir por el vehículo aerodeslizador. El

radio del círculo es $p$ y la frecuencia de oscilación es $w$. De interés es el valor de $\beta$, que se escogió según el encontrado experimentalmente para el vehículo de la figura 1. Los otros valores se utilizan para determinar las matrices $Q$ y $R$ de la función de costo para la ganancia de control $K$. El tiempo de muestreo es $t_{s}$, y $\rho$ es un coeficiente de peso que se utiliza para añadir o reducir importancia a la parte dinámica o la parte de actuación en la función de costo.

La trayectoria a la cual navegará el vehículo se muestra en la figura 4. Esta es una trayectoria circular a pesar de como se pueda ver por la diferencia en la escala de los ejes del gráfico. La figura 4 muestra tres ilustraciones, arriba se grafica la posición del vehículo según la coordenada $y$ del sistema de referencia contra su posición en $X$, cada par de puntos comparten el mismo valor de tiempo. En las dos ilustraciones de abajo se grafican cada coordenada deseada con respecto al tiempo, como es de esperar estas gráficas son sinusoidales según esta variable independiente.

Para estos parámetros el vector de ganancia del controlador queda completamente definido. Al utilizar la función $l q r$ de MATLAB con las matrices $A, B, Q$ y $R$ que se presentan aquí el vector de ganancias es el que se

presenta en la ecuación (18).

$$
K=\left[\begin{array}{c}
92.2877 \\
-472.3233 \\
49.2097 \\
-108.9291
\end{array}\right]^{T}
$$

El origen del eje coordenado del sistema de referencia se determina como la posición de la nave al momento en que se inicia la simulación. La simulación se diseñó para que se pudieran indicar varias condiciones iniciales diferentes, pudiendo probar posiciones lejos del centro del círculo, o con ciertas velocidades iniciales. La sintonización de las matrices $\mathrm{R}$ y $\mathrm{Q}$ se llevó a cabo variando estas condiciones iniciales, la intención era lograr que para un grupo predeterminado de condiciones iniciales el sistema se estabilizara en menos de 60 segundos.

Tabla 1. Valores para la simulación del aerodeslizador

\begin{tabular}{|l|l|l|l|}
\hline Variable & \multicolumn{1}{|c|}{ Valor } & \multicolumn{1}{c|}{ Variable } & Valor \\
\hline$p$ & $5 \mathrm{~m}$ & $x_{2 \max }$ & 0.1 \\
\hline$w$ & $\pi / 16 \mathrm{rad} / \mathrm{s}$ & $x_{3 \max }$ & $1.0 \mathrm{e}-5$ \\
\hline$\beta$ & $347 / 30001 / \mathrm{s}$ & $x_{4 \max }$ & $1.0 \mathrm{e}-3$ \\
\hline$u_{1 \max }$ & 0.1 & $t_{s}$ & $0.1 \mathrm{~s}$ \\
\hline$x_{1 \max }$ & 0.01 & $\rho$ & $0.7 \mathrm{e} 5$ \\
\hline
\end{tabular}



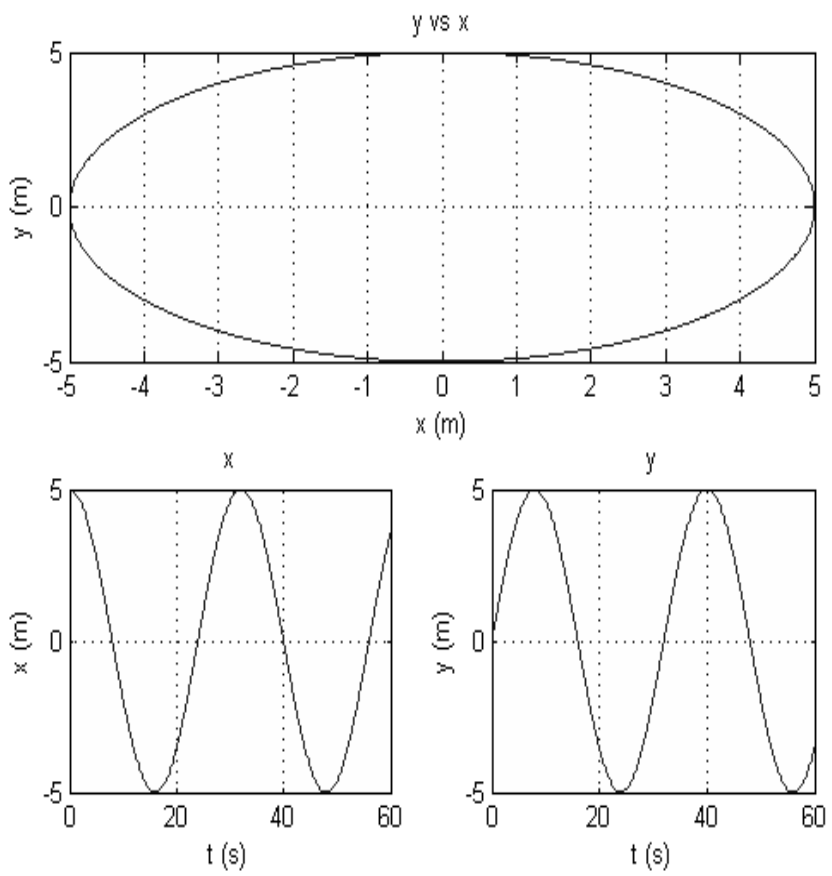

Figura 4. Trayectoria deseada para la navegación del aerodeslizador.

\section{Resultados de la simulación}

La respuesta simulada con MATLAB frente a diversas condiciones iniciales se muestra en las siguientes imágenes. El vector de condiciones iniciales es $X i=\left[\begin{array}{llll}u_{i} & v_{i} & Z_{1 i} & Z_{2 i}\end{array}\right]$, donde las variables tienen los significados usuales expresados en las secciones anteriores. Así si se desea que la simulación inicie con una velocidad inicial de $50 \mathrm{~m} / \mathrm{s}$ en la dirección de estribor-babor, se usaría el vector $\left[\begin{array}{llll}0 & -50 & 0 & 0\end{array}\right]$. Se presentan los resultados de la simulación para dos vectores de condiciones iniciales. La respuesta ante el vector de reposo $\left[\begin{array}{llll}0 & 0 & 0 & 0\end{array}\right]$ donde el vehículo inicia su movimiento en el centro del eje coordenado y luego es actuado para seguir la trayectoria de referencia. El resultado de esta simulación se muestra en la figura 5. La figura muestra cinco imágenes, a mano izquierda la respuesta geométrica de la posición del vehículo, y a mano derecha las cuatro respuestas gráficas de las variables de estado. La respuesta geométrica de la posición del vehículo es la posición $x$ y $y$, graficadas como un par ordenado para cada instante de tiempo que compartan; se obtiene a partir de las variables de estado $\begin{array}{lllllll}Z_{1} & \text { y } & Z_{2} & \text { a las cuales se les vuelve a aplicar el }\end{array}$ difeomorfismo para obtener la posición según el eje de referencia. El aro exterior es la trayectoria de referencia, la línea solida interna es la posición del vehículo simulado en cada instante. Se observa que el vehículo nunca se aleja más allá de cinco metros de la trayectoria deseada. Otro detalle interesante es la curva pronunciada que se puede observar en el cuadrante superior izquierdo, donde aparentemente la señal de actuación invierte su dirección. Al lado derecho se observan las respuestas de las variables de estado, las dos de arriba: las velocidades, a la izquierda la velocidad popa-proa y a mano derecha la velocidad babor-estribor, se observa que ambas velocidades se asientan en un tiempo no mayor a 40 segundos. Las dos variables de estado de abajo $Z_{1}$ y $Z_{2}$ se relacionan directamente con la posición y se espera que se asienten a un valor que depende del tamaño del círculo y la frecuencia de oscilación del vehículo. Se nota que se estabilizan en 40 segundos de igual modo.

También se simuló la respuesta a un caso crítico, se desea saber como reaccionaría el vehículo ante una condición de velocidad inicial. La idea es comprobar cuál sería la reacción del vehículo cuando este deba asumir una nueva consigna repentinamente durante su operación. Para un vehículo aerodeslizador pequeño y liviano que no está diseñado para ser veloz, una velocidad de trabajo puede rondar los $10 \mathrm{~m} / \mathrm{s}$, así que se utiliza este valor como punto inicial en las velocidades $u$ y $v$. La figura 6 muestra la respuesta del sistema para las condiciones iniciales $\left[\begin{array}{llll}10 & 10 & 0 & 0\end{array}\right]$. Se observa en la respuesta de posición que el vehículo se aleja de la consigna más de 25 metros, no obstante a mano izquierda se puede verificar que la condición de tiempo de asentamiento se cumple aparentemente al mismo tiempo que en el caso donde se partió del reposo. Aunque puede parecer alarmante, una diferencia con la referencia tan grande, al recordar que este vehículo se ha diseñado para aplicaciones agrícolas, donde los campos pueden extenderse por hectáreas, 25 metros no parece una cantidad muy grande.

\section{Conclusiones y trabajo futuro}

Se ha probado con una simulación que, para una trayectoria circular y mediante la aplicación de un difeomorfismo, es posible controlar el sistema del aerodeslizador para que navegue una trayectoria circular por medio de un controlador basado en LQR. 

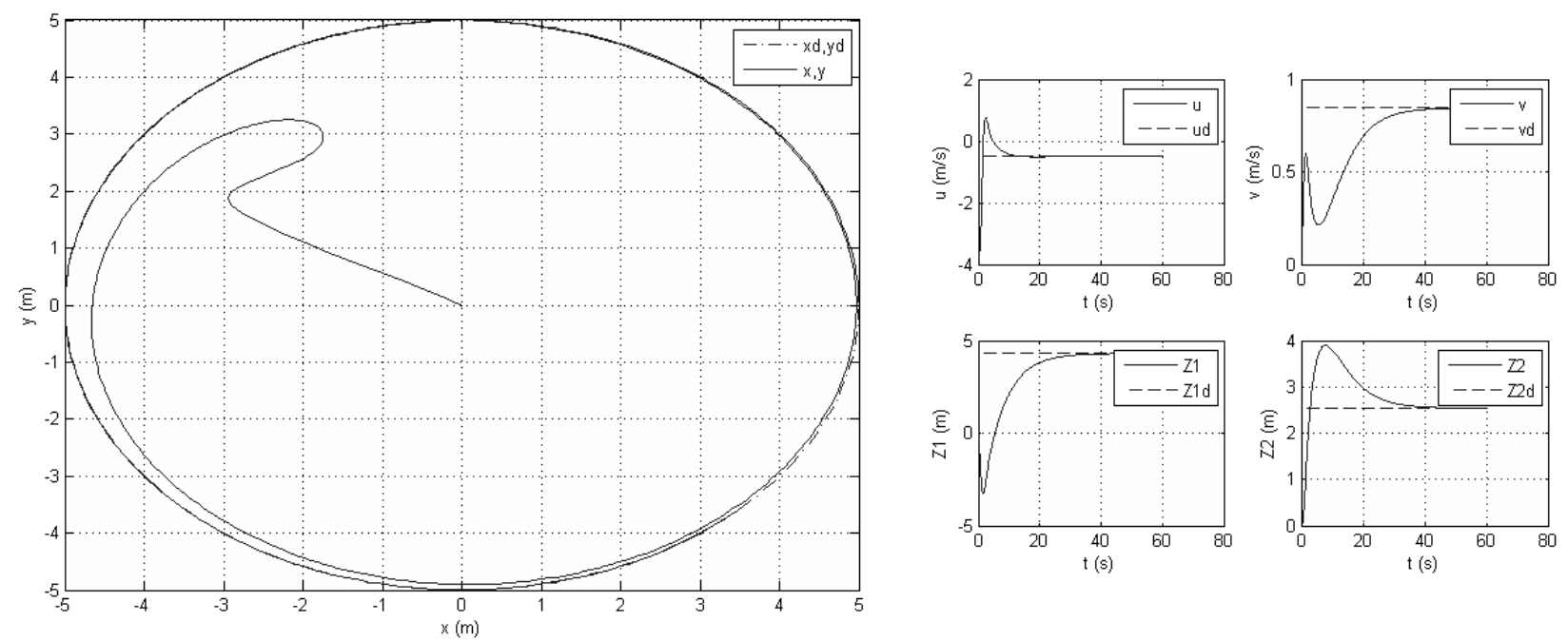

Figura 5. Respuesta del controlador LQR para una condición inicial $\left[\begin{array}{cccc}0 & 0 & 0 & 0\end{array}\right]$.
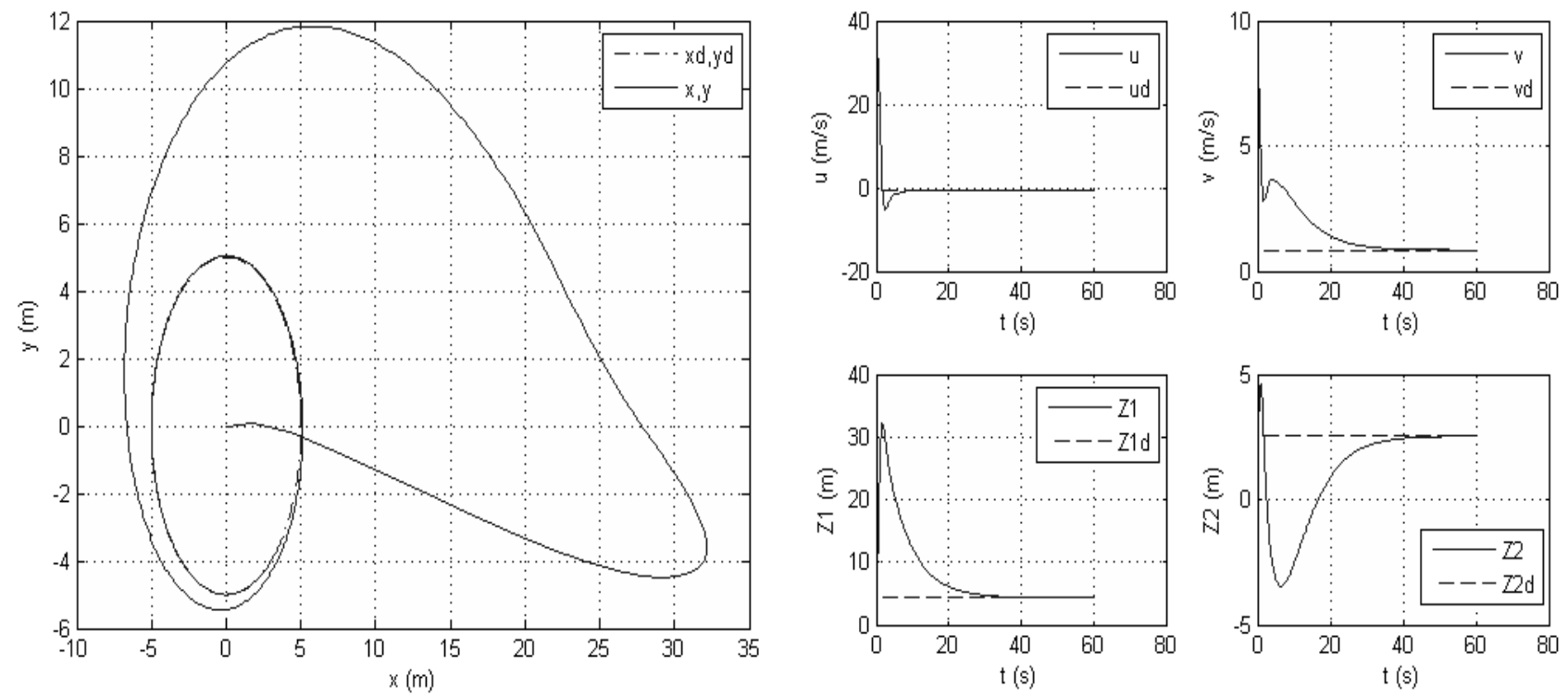

Figura 6. Respuesta del controlador LQR para una condición inicial $\left[\begin{array}{llll}10 & 0 & 0 & 0\end{array}\right]$.

Por ende se ha presentado como novedad en el arte un controlador que logra que un aerodeslizador no tripulado y autónomo siga una trayectoria circular usando un único actuador en la dirección de avance.

El controlador tiene espacio para mejorar, los siguientes pasos de este estudio involucran la implementación del controlador en sistemas físicos y su subsecuente caracterización para su funcionamiento eficaz. Otro punto interesante para el estudio concierne a la señal de actuación, un estudio profundo de cómo evoluciona con diferentes parámetros podría ayudar a mejor el rendimiento del controlador. 


\section{Referencias}

[1] NRCS (NATURAL RESOURCES CONSERVATION SERVICE), «Precision Agriculture: NRCS Support for Emerging Technologies,» United States Department of Agriculture, 2007. [En línea]. Available: https://www.nrcs.usda.gov/Internet/FSE_DOCUMENTS/stelprdb10 43474.pdf. [Último acceso: 27 Diciembre 2018].

[2] A. Lavers, «Guidelines on Good Practice for Aerial Application of Pesticides,» Food and Agriculture Organization of the United Nations, 2001. [En línea]. Available: http://www.fao.org/docrep/006/y2766e/y2766e00.htm. [Último acceso: 7 Julio 2018].

[3] A. Barton, «Vickers Armstrong hovercraft of the early 1960s,» 2018. [En línea]. Available: https://www.bartiesworld.co.uk/hovercraft/vickers.htm. [Último acceso: 2018 Diciembre 27].

[4] V. V. Rumyantsev, «Non-holonomic systems,» Encyclopedia of Mathematics, 7 Febrero 2011. [En línea]. Available: http://www.encyclopediaofmath.org/index.php?title=Non-

holonomic_systems\&oldid=12986. [Último acceso: 28 Diciembre 2018].

[5] R. Teldrake, «6.832 Underactuated Robotics. Chapter 1.,» Primavera 2009. [En línea]. Available: https://ocw.mit.edu/courses/electrical-engineering-and-computerscience/6-832-underactuated-robotics-spring2009/readings/MIT6_832s09_read_ch01.pdf. [Último acceso: 28 Diciembre 2018].
[6] D. García, «Dynamic, Simulation and Control Design of an Unmanned Hovercraft,»I+D Tecnológico, vol. 10, $\mathrm{n}^{\circ}$ 2, pp. 40-47, 2016.

[7] H. Sira-Ramirez y C. A. Ibanez, «The control of the hovercraft system: a flatness based approach,» de Proceedings of the 2000. IEEE International Conference on Control Applications. Conference Proceedings (Cat. No.00CH37162), Anchorage, AK, USA, 2000.

[8] D. García y W. N. White, «Nonlinear Control of an Unmanned Hovercraft for Agricultural Applications,» de ASABE CSBE/SGCAB Annual Meeting, Montreal, Canada, 2014.

[9] I. Fantoni y R. Lozano, «The Hovercraft Model,» de Non-Linear Control for Underactuated Mechanical Systems, London, Springer, 2002, pp. 155-171.

[10] A. Tewari, «Linear Optimal Control,» de Modern Control Design with Matlab and Simulink, Chichester, John Wiley \& Sons, 2002, pp. 283-321.

[11] R. M. Murray, «CDS 110b. Clase, Tema: Lecture 2 - LQR Controller,» California Institute Of Technology, California, enero 11, 2006. 